DOI:

10.1038/nphys869
MILESTONE 14

\section{Sticking together}

The fact that a dirty metal can support an electric current flowing without resistance might sound exotic to some, but it is a textbook property of 'conventional' superconductors. When spins are involved, however, the superconductors do become 'exotic'.

The conventional mechanism of superconductivity was explained by John Bardeen, Leon Cooper and Robert Schrieffer (in what is known as the BCS theory) back in 1957. For decades, it was a mystery how electrons, which are classified as 'fermions', could be forced into a single ground state that was more typical for 'bosons' (as when they undergo Bose-Einstein condensation). Fermions cannot all pile into the same ground state because only two - one with its spin pointing upwards and the other pointing downwards - can occupy each quantum state; bosons do not heed such conventions. As it turns out, the only way for fermions to form a condensate is for them to pair up, with the help of the crystalline lattice: when one electron passes through the lattice, the positive ions are slightly attracted to the passing negative electron; if a second electron comes along, it will sense the deformed lattice and be attracted to the net positive charge, and hence to the original electron. This kind of lattice-assisted coupling is weak, but it is strong enough for the paired electrons to drop down to a collective ground state.

Naturally, people considered whether such pairing could glue other fermions together, in particular ${ }^{3} \mathrm{He}$. As ${ }^{4} \mathrm{He}$ exhibits superfluidity - that is, the liquid can flow without viscosity below a certain temperature - it was believed (hoped) that ${ }^{3} \mathrm{He}$ would do likewise. Yet, given that ${ }^{4} \mathrm{He}$ is a boson and ${ }^{3} \mathrm{He}$ is a fermion, it was not clear how ${ }^{3} \mathrm{He}$ could condense until the BCS theory came along. However, the magnetic interactions between the ${ }^{3} \mathrm{He}$ particles are strong, and so they cannot pair up as electrons do. Instead, the pairing glue must come from another source.

The first authors to propose ferromagnetic fluctuations of the spins as such a glue were A. Layzer and D. Fay. When one particle whizzes through the liquid, its spin (pointing upwards, for instance) attracts other spin-up particles and repels spin-down particles. The effective spin-up polarization can then attract another spin-up particle, leading to a spin-up-spinup pair. When coupled in this way, the ${ }^{3} \mathrm{He}$ atoms are able to form a superfluid.

Amazingly, all the theoretical groundwork, including that by Philip Anderson and Pierre Morel, and by Roger Balian and Richard Werthamer, was laid down before the experimental confirmation of a superfluid state in ${ }^{3} \mathrm{He}$ — that came in 1972 and earned its authors, Douglas Osheroff, Robert Richardson and David Lee, the Nobel prize in Physics in 1996.

Although superfluidity was anticipated, measurements revealed several unique superfluid phases in ${ }^{3} \mathrm{He}$. Moreover, because of the non-zero spin of the pairs (in conventional superconductors the net spin is zero), ${ }^{3} \mathrm{He}$ also yielded some unexpected properties. In 1987, a team working in Moscow discovered a pure spin supercurrent. Unlike the supercurrent in a conventional

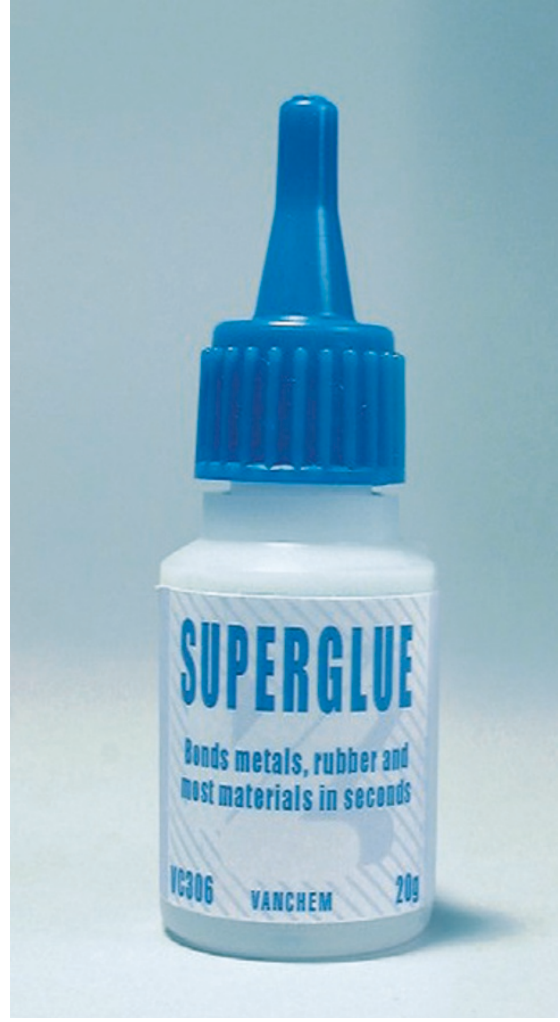

superconductor that carries charge and mass, the spin supercurrent carries only spin and there is no mass flow. ${ }^{3} \mathrm{He}$ is truly exotic, because of its spin.

May Chiao, Senior Editor, Nature Physics

ORIGINAL RESEARCH PAPERS Bardeen, J., Cooper, L. N. \& Schrieffer, J. R. Microscopic theory of superconductivity. Phys. Rev. 106, 162-164 (1957)|Anderson, P. W. \& Morel, P. Generalized Bardeen-Cooper-Schrieffer states and the proposed low-temperature phase of liquid $\mathrm{He}^{3}$. Phys. Rev. 123, 1911-1934 (1961)| Balian, R. \& Werthamer, N. R. Superconductivity with pairs in a relative $p$ wave. Phys. Rev. 131,1553-1564 (1963) | Layzer, A. \& Fay, D. Superconducting pairing tendency in nearly ferromagnetic systems. Int. J. Magn. 1, 135-141 (1971)|Osheroff, D. D., Richardson, R. C. \& Lee, D. M. Evidence for a new phase of solid He $\mathrm{H}^{3}$. Phys. Rev. Lett. 28, 885-888 (1972) | Osheroff, D. D., Gully, W. J., Richardson, R. C. \& Lee, D. M. New magnetic phenomena in liquid $\mathrm{He}^{3}$ below $3 \mathrm{mK}$. Phys. Rev. Lett. 29, 920-923 (1972) | Borovik-Romanov, A. S. Bun'kov, Yu. M., Dmitriev, V. V. \& Mukharskii, Yu. M. Observation of phase slippage during the flow of a superfluid spin current in ${ }^{3} \mathrm{He}$-B. JETP Lett. 45, 124-128 (1987)

FURTHER READING Leggett, A. J. A theoretical description of the new phases of liquid ${ }^{3} \mathrm{He}$ Rev. Mod. Phys. 47, 331-414 (1975) 